\title{
Production of dileptons via photon-photon fusion in proton-proton collisions with one forward proton measurement
}

\author{
Antoni Szczurek, ${ }^{a, b, *}$ Barbara Linek $^{b}$ and Marta Łuszczak $^{b}$ \\ ${ }^{a}$ Institute of Nuclear Physics, \\ ul. Radzikowskiego 152, PL-31-342 Kraków, Poland \\ ${ }^{b}$ College of Natural Sciences, Institute of Physics, \\ ul. Pigonia 1, PL-35-959 Rzeszów, Poland
}

E-mail: antoni.szczurek@ifj.edu.pl

We discuss the mechanism of dilepton production in proton-proton scattering via fusion of virtual photons with identification of one proton on either side. This is relevant for the ATLAS+AFP and CMS+PPS at the LHC. Transverse momenta of the photons are taken into account via photon unintegrated fluxes. The latter ones are expressed in terms of proton electromagnetic form factors and structure functions. We include different categories of such processes (double elastic, single dissociative). Some differential distributions are shown explicitly and differences with respect to the results without proton identification are discussed. A soft gap survival factor is calculated using SuperChic-4 code.

*** The European Physical Society Conference on High Energy Physics (EPS-HEP2021), ***

*** 26-30 July $2021 * * *$

*** Online conference, jointly organized by Universität Hamburg and the research center DESY ***

\footnotetext{
${ }^{*}$ Speaker
} 

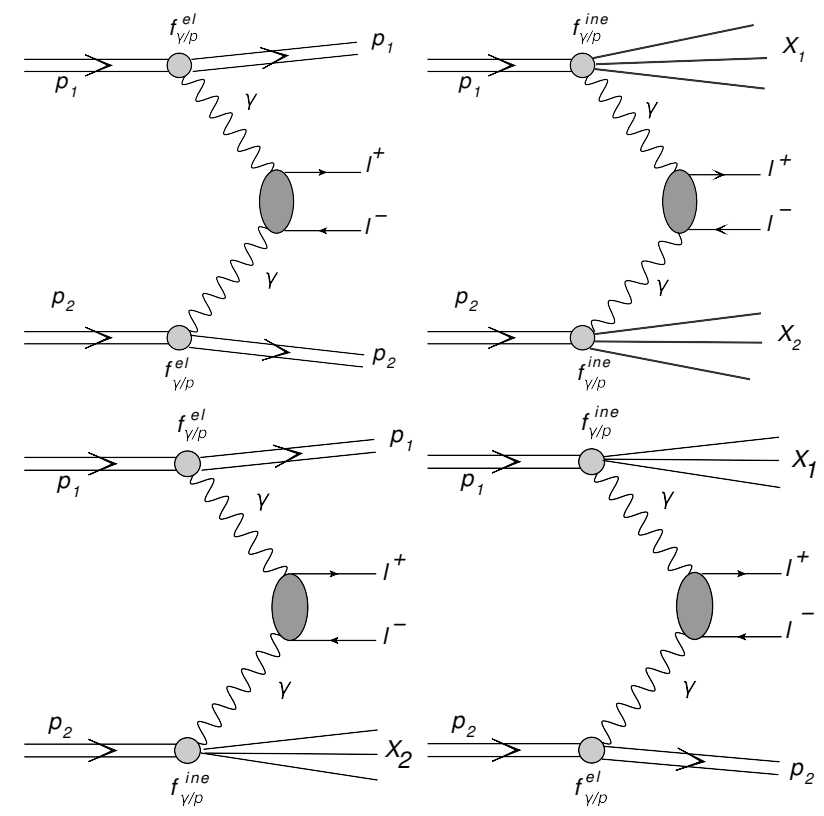

Figure 1: Four different categories of $\gamma \gamma$ fusion mechanisms of dilepton production in proton-proton collisions.

\section{Introduction}

There are many mechanisms of dilepton production in proton-proton collisions. One of them is photon-photon fusion mechanism. So far such a mechanism was studied by measuring dileptons and imposing a condition on rapidity gaps around dileptons. Recently the CMS [1] and ATLAS [2] collaborations presented results for the case when one proton is measured in forward direction. We have developed a code which can calculate such processes [3-5]. In order to compare the theoretical results to the experimental data one has to impose kinematical condition on so-called $\xi$-variable $[1,2]$.

Here we show results based on our recent paper [6]. In our calculations we use the formalism developed by our group in [3-5]. How to include rapidity gap survival factor related to emission of (mini)jets was disussed in [7, 8] for $W^{+} W^{-}$and $t \bar{t}$ production. In [6] we repeated similar analysis also for $\mu^{+} \mu^{-}$production. The absorption for double-elastic contribution was studied e.g. in [9, 10] within a momentum space formalism. The impact parameter approach can be found in [11].

\section{Sketch of the formalism}

There are four categories of the $\gamma \gamma$ processes as shown in Fig.1. We call them elastic-elastic, inelastic-inelastic, elastic-inelastic and inelastic-elastic. The double inelastic contribution is not included when proton is measured (as there no explicit proton appears in the formalism).

In the $k_{T}$-factorization approach [3,4], the cross section for production of $l^{+} l^{-}$can be written 


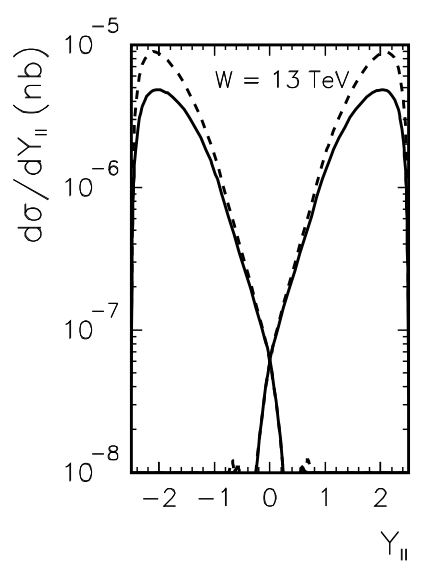

Figure 2: Distribution in dilepton rapidity for four different contributions considered. Here the cuts on $\xi_{l l}^{+}$or $\xi_{l l}^{-}$are imposed. The solid line is for double elastic contribution and the dashed line is for single dissociation contribution.

in the form

$$
\frac{d \sigma^{(i, j)}}{d y_{1} d y_{2} d^{2} \boldsymbol{p}_{1} d^{2} \boldsymbol{p}_{2}}=\int \frac{d^{2} \boldsymbol{q}_{1}}{\pi \boldsymbol{q}_{1}^{2}} \frac{d^{2} \boldsymbol{q}_{2}}{\pi \boldsymbol{q}_{2}^{2}} \mathcal{F}_{\gamma^{*} / A}^{(i)}\left(x_{1}, \boldsymbol{q}_{1}\right) \mathcal{F}_{\gamma^{*} / B}^{(j)}\left(x_{2}, \boldsymbol{q}_{2}\right) \frac{d \sigma^{*}\left(p_{1}, p_{2} ; \boldsymbol{q}_{1}, \boldsymbol{q}_{2}\right)}{d y_{1} d y_{2} d^{2} \boldsymbol{p}_{1} d^{2} \boldsymbol{p}_{2}},
$$

where the indices $i, j \in\{\mathrm{el}$, in $\}$ denote elastic or inelastic final states. Here the photon flux for inelastic case is integrated over the mass of the remnant.

The ATLAS collaboration analysis imposes a special condition on:

$$
\xi_{1}=\xi_{l l}^{+}, \xi_{2}=\xi_{l l}^{-}
$$

The longitudinal momentum fractions of the photons were calculated in the ATLAS analysis as:

$$
\begin{aligned}
& \xi_{l l}^{+}=\left(M_{l l} / \sqrt{s}\right) \exp \left(+Y_{l l}\right), \\
& \xi_{l l}^{-}=\left(M_{l l} / \sqrt{s}\right) \exp \left(-Y_{l l}\right) .
\end{aligned}
$$

Only lepton variables enter the formula.

\section{Results}

As an example in Fig.2 we show a distribution in $Y_{l l}$ (rapidity of the $l^{+} l^{-}$system). One can observe a deep dip at $Y_{l l} \approx 0$ which is due to the imposed cuts on the $\xi$-variable. When the cuts are removed the dip is not present [6].

What are typical $x_{B j}$ and $Q^{2}$, i.e. arguments of the structure functions for the considered processes with single proton dissociation, is shown in Fig.3. Both perturbative $\left(Q^{2}>2 \mathrm{GeV}^{2}\right)$ and nonperturbative $\left(Q^{2}<2 \mathrm{GeV}^{2}\right)$ regions enter the corresponding calculations. The nonperturbative 

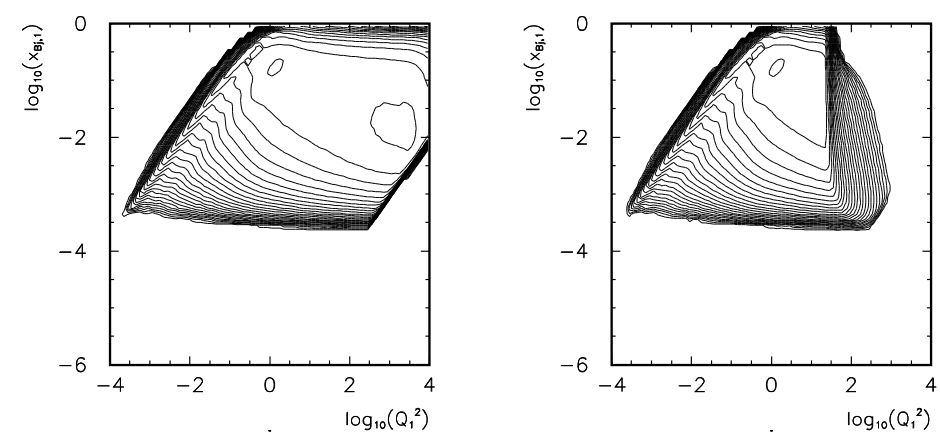

Figure 3: The range in the $\left(x_{B j}, Q^{2}\right)$ space tested in inelastic-elastic (left) with $\xi$ cuts. The right panel includes also an extra cut $p_{t, \text { pair }}<5 \mathrm{GeV}$ as imposed by the ATLAS collaboration [2].

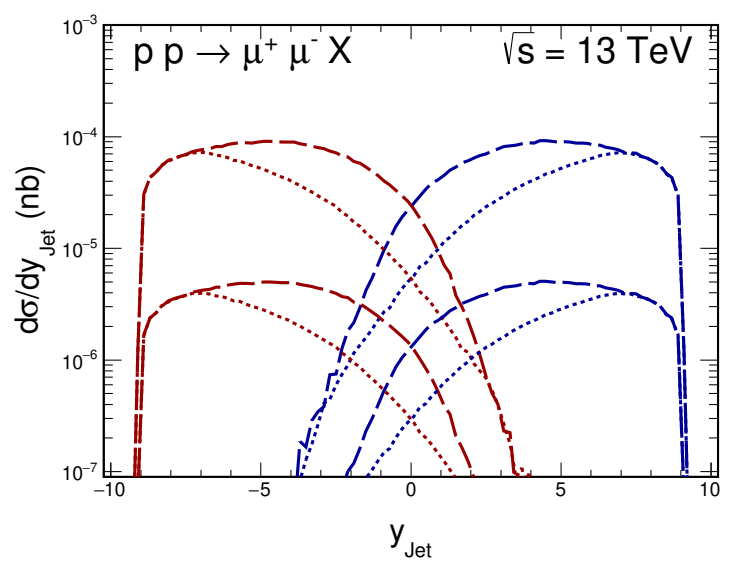

Figure 4: Distribution in rapidity of (mini)jets for inclusive case (upper curves) and for the case with cut on $\xi_{1 / 2}$ (lower curves). The extra dotted lines represent results that include the cut $p_{t, p a i r}<5 \mathrm{GeV}$ as in the ATLAS analysis [2].

region is even relatively larger when the cut on $p_{t, \text { pair }}<5 \mathrm{GeV}$ is imposed as in the recent ATLAS [2] paper.

Many other distributions were discussed in [6]. The emission of photon coupled to quarks/antiquarks (single dissociative process) may lead to a production of a extra minijet. Such s minijet leads to a distroying rapidity gap and lowering the corresponding cross sections with gap condition. The rapidity distribution of such jets is shown in Fig.4.

Now we wish to show some results obtained using the SuperChic-4 generator [12].

In Fig. 5 we show corresponding gap survival factor calculated as:

$$
S_{G}\left(Y_{l l}\right)=\frac{d \sigma /\left.d Y_{l l}\right|_{\text {withSR }}}{d \sigma /\left.d Y_{l l}\right|_{\text {without } S R}}
$$

as a function of $Y_{l l}$ variable.

Without the $\xi$ cut we observe quite different shapes of distributions in $Y_{l l}$ without and with soft rapidity gap survival factor (see the left panel). When the $\xi$-cut is imposed the distributions 

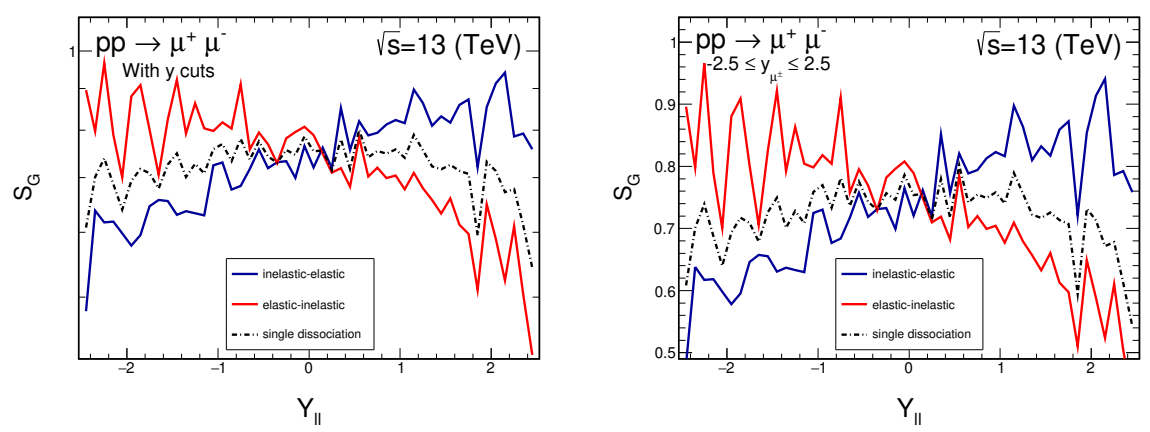

Figure 5: The soft gap survival factor as a function of rapidity of the $\mu^{+} \mu^{-}$pair for single proton dissociation. We show the result without $\xi$ cuts (left panel) and with $\xi$ cuts (right panel). The dash-dotted black line represents effective gap survival factor for both single-dissociation components added together.
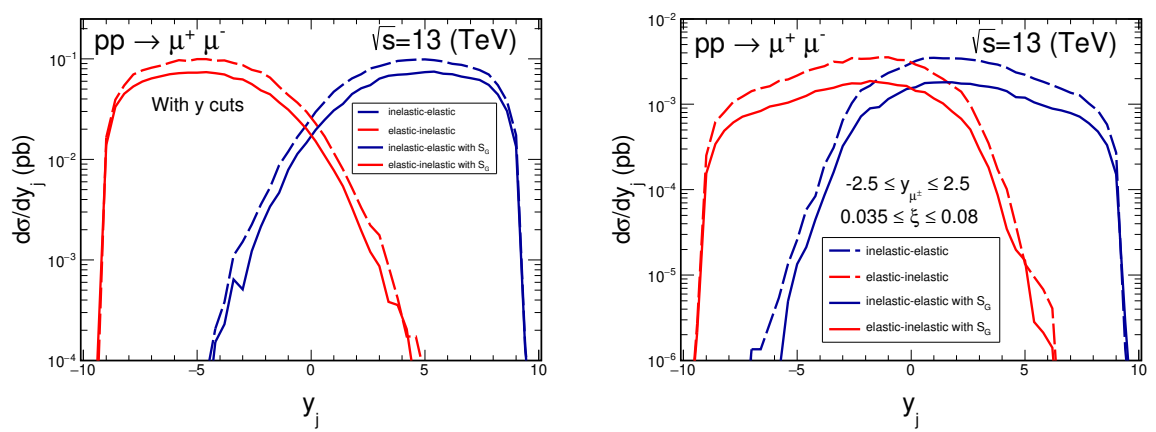

Figure 6: Distribution in the (mini)jet rapidity for the inclusive case with no $\xi$ cut (left panel) and when the cut on $\xi$ is imposed (right panel) for elastic-inelastic and inelastic-elastic contributions as obtained from the SuperChic-4 generator. We show result without (dashed line) and with (solid line) soft rescattering correction.

with and without soft rapidity gap survival factor have very similar shapes. Then, however, the elastic-inelastic and inelastic-elastic contributions are well separated in $Y_{l l}$.

In Fig. 6 we show the (mini)jet distribution in rapidity for elastic-inelastic and inelastic-elastic components. We show the distribution without imposing the $\xi$ cut (left panel) and when imposing the $\xi$ cut (right panel). One can observe slightly different shape for both cases. The corresponding gap survival factor (probability of no jet in the main detector) is 0.8 and 0.5 , respectively.

\section{Conclusions}

In this proceedings we have reported some selected results of our recent studies of $\mu^{+} \mu^{-}$ production in proton-proton scattering associated with the emission of one forward proton. This was accomplished by imposing a cut on proton energy loss fraction. We have included both double elastic and single dissociative contribution. The double-dissociative contribution was ignored as 
here there is no explicit proton. In our recent paper [6] we considered both continuum production and production of $\Delta^{+}$isobar and other resonances.

Several distributions were discussed in [6]. Here we have shown only some selected results. Some difference compared to the case of no proton registration conditions have been pointed out. Particularly interesting is the distribution in $Y_{l l}$ which has a deep minimum at $Y_{l l} \sim 0$. The minimum at $Y_{l l}=0$ is caused by the application of a condition on $\xi_{l l}^{ \pm}$imposed on the leading proton.

For comparison we have shown results of calculation using popular SuperChic-4 generator. In general, the results are very similar to those obtained with our codes. We have shown also some results for kinematics-dependent gap survival factor. We have found some interesting dependence of gap survival factor on $Y_{l l}$. Finally we have shown rapidity distribution of a (mini)jet associated with partonic processes, also when including soft rescattering corrections.

\section{Acknowledgements}

This study was partially supported by the Polish National Science Center grant UMO-2018/31/B/ST2/03537 and by the Center for Innovation and Transfer of Natural Sciences and Engineering Knowledge in Rzeszów.

\section{References}

[1] A.M. Sirunyan et al. (CMS Collaboration), "Observation of proton-tagged, central (semi)exclusive production of high-mass lepton pairs in $\mathrm{p} \mathrm{p}$ collisions at $13 \mathrm{TeV}$ with the CMS-TOTEM precision proton spectrometer", JHEP07 (2018) 153.

[2] G. Aad et al. (ATLAS collaboration), "Observation and measurement of forward proton scattering in association with lepton pairs produced via the photon fusion mechanisms at ATLAS”, Phys. Rev. Lett. 125, 261801 (2020), arXiv:2009.14537.

[3] G.G. da Silveira, L. Forthomme, K. Piotrzkowski, W. Schäfer and A. Szczurek, "Central $\mu^{+} \mu^{-}$ production via photon-photon fusion in proton-proton collisions with proton dissociation", JHEP 02 (2015) 159.

[4] M. Łuszczak, W. Schäfer and A. Szczurek, "Two-photon dilepton production in proton-proton collisions: Two alternative approaches”, Phys. Rev. D93 (2016) 074018.

[5] M. Łuszczak, W. Schäfer and A. Szczurek, "Production of $W^{+} W^{-}$pairs via $\gamma^{*} \gamma^{*} \rightarrow W^{+} W^{-}$ subprocess with photon transverse momenta", JHEP05 (2018) 064.

[6] A. Szczurek, B. Linek and M. Łuszczak, "Semiexclusive dilepton production in proton-proton collisions with one forward proton measurement at the LHC”, Phys. Rev. D104 074009 (2021), arXiv:2107.02535.

[7] L. Forthomme, M. Luszczak, W. Schäfer and A. Szczurek, "Rapidity gap survival factors caused by remnant fragmentation for $W^{+} W^{-}$pair production via $\gamma^{*} \gamma^{*} \rightarrow W^{+} W^{-}$subprocess with photon transverse momenta", Phys. Lett. B789 (2019) 300 . 
[8] M. Luszczak, L. Forthomme, W. Schäfer and A. Szczurek,

"Production of $t \bar{t}$ pairs via $\gamma \gamma$ fusion with photon transverse momenta and proton dissociation", JHEP 02 (2019) 100.

[9] P. Lebiedowicz and A. Szczurek, "Exclusive production of heavy charged Higgs boson pairs in the $p p \rightarrow p p H^{+} H^{-}$reaction at the LHC and a future circular collider", Phys. Rev. D91 (2015) 095008.

[10] P. Lebiedowicz and A. Szczurek, "Exclusive and semiexclusive production of $\mu^{+} \mu^{-}$pairs with Delta isobars and other resonances in the final state and the size of absorption effects", Phys. Rev. D98 (2018) 053007.

[11] M. Dyndal and L. Schoeffel, "The role of finite-size effects on the spectrum of equivalent photons in proton-proton collisions at the LHC", Phys. Lett. B741 (2015) 66.

[12] L.A. Harland-Lang, M. Tasevsky, V.A. Khoze and M.G. Ryskin, "A new approach to modelling elastic and inelastic photon-initiated production at the LHC: SuperChic 4", Eur. Phys. C80 (2020) 925. 